\title{
OFF-DISK IMPLANTATION OF EARLY SOLAR WIND INTO A PLANETESIMAL-DUST CLOUD
}

\author{
SHO SASAKI \\ Institute of Geology and Mineralogy \\ Faculty of Science \\ Hiroshima University \\ Hiroshima 790 \\ Japan
}

ABSTRACT. Off-disk implantation of ancient solar wind into a protoplanetary dust cloud can explain the present amounts of solar-type noble gases in gas-rich meteorites and Venus, even if the dust cloud is very opaque along its midplane.

\section{Introduction}

The high abundances of solar-type noble gas in lunar soils and some air-borne particles are considered to come from the implantation of present solar wind. In the present solar system, noble gases with solar-type elemental and isotopic abundances are also found in gas-rich meteorites (Goswani et al., 1984) and Venus' atmosphere (Pollack and Black, 1982). Furthermore, He and Ne in terrestrial mantle show solar-type isotopic signatures. The implantation of ancient solar wind into a protoplanetary dust cloud is a possible mechanism to explain these ubiquitous solar-type noble gases (Wetherill, 1981; McElroy and Prather, 1981).

Although the remnant grains of initial condensates dissipated along with the solar nebula, dust grains were produced by planetesimal collisions (Nakano, 1988). The opacity of the dust swarm is proportional to dust numbcr density and inverscly proportional to dust size. If the dust cloud was opaque and optical thickness along the disk midplane was high, solar wind species would be trapped only at the inner edge (0.05-0.1AU) of the disk (Wetherill, 1981; Prinn and Fegley, 1989) (see Fig. 1(a)). If the dust cloud was transparent, the solar wind would penetrate but no significant implantation would take place. Then the implantation of a large amount of the ancient solar wind flux did not occur ubiquitously in the solar system?

The dust swarm should have a vertical distribution, reflecting orbital inclinations of dust-forming planetesimals. Even if optical thickness along the disk midplane is 
very large, the solar wind as well as radiation can penetrate through the off-disk region of the disk (Sasaki, 1989; 1990) (see Fig. 1 (b)). In the present report, we estimate trapped amounts of solar-wind noble gases, taking into account a vertical distribution of dust grains.

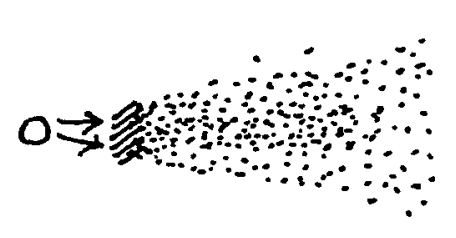

(a)

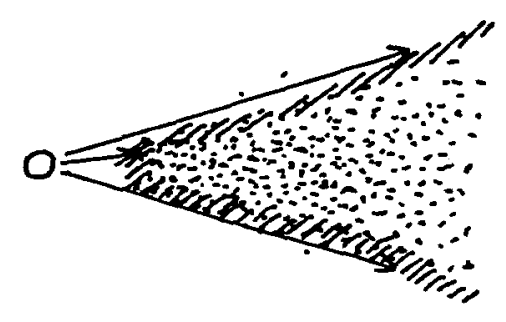

(b)

Fig. 1 Schematic picture of solar wind implantation: (a) Inner boundary implantation (b) Off-disk implantation

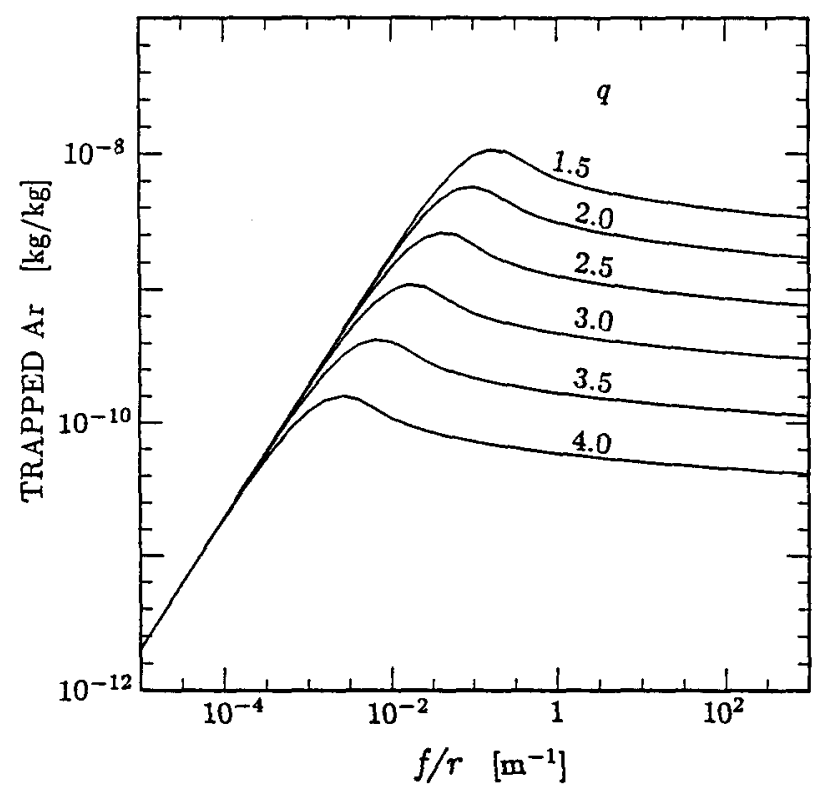

Fig. 2 Total trapped amount of ${ }^{36} \mathrm{Ar}$ between 3 and $3.5 \mathrm{AU}$. The values are given by $\mathrm{kg} \mathrm{Ar}$ per $\mathrm{kg}$ total solid mass in the region. The horizontal axis is $f / r\left[\mathrm{~m}^{-1}\right]$. Solar wind intensity is chosen to be 100 times the present value and implantation duration is assumed to be $10^{7} \mathrm{yr}$. 


\section{Dust Distribution}

The dust cloud is assumed to have a power-law density distribution radially and a gaussian distribution vertically. We assume that the disk is truncatcd at $a_{0}=$ $0.1[\mathrm{AU}]$ by dust evaporation. We have mass distribution of dust

$$
\rho_{\text {dust }}(a, z)=\rho_{\text {de }} f\left(\frac{a}{1 \mathrm{AU}}\right)^{-q} \exp \left\{-\left(\frac{z}{h}\right)^{2}\right\},
$$

where $f$ is mass fraction of dust relative to the total solid mass and $\rho_{\text {de }} f$ expresses the dust mass abundance at the midplane of the terrestrial region $(a=1[\mathrm{AU}]$ and $z=0$ ). Using the average orbital inclination of dust $i$, the vertical scale height $h$ is written by $h=i a$. We have $i=0.01$ from random velocities of planetesimals in the late stage of planetary formation. This inclination gives $\rho_{d e}=2.7 \times 10^{-8}\left[\mathrm{~kg} / \mathrm{m}^{3}\right]$ with the present smeared solid mass distribution (Hayashi, et al., 1985). Since the opacity determines the solar wind implantation, the ratio of dust mass fraction to dust size $f / r$ is an important parameter controlling our results.

\section{Results}

We obtain integrated amounts of solar wind ${ }^{36} \mathrm{Ar}$ in a vertical column. Figure 2 shows the trapped amount of solar wind ${ }^{36} \mathrm{Ar}$ between 3.0 and $3.5 \mathrm{\Lambda U}$. Solar wind intensity is assumed 100 times larger than the present value, and implantation duration is assumed to be $10^{7} \mathrm{yr}$. When $f / r$ is small. disk is so transparent that efficient wind capture does not occur. When $f / r$ is large $\left(>10^{-1}\left[\mathrm{~m}^{-1}\right]\right)$, the solar wind implantation takes place in the off-disk region. Because larger $f / r$ simply moves trapping region vertically outward, the total trapped amount does not decrease largely (Sasaki, 1990). Gas-rich meteorites with high noble gas concentration have ${ }^{36} \mathrm{Ar}$ abundances around $10^{-3}[\mathrm{~kg} / \mathrm{kg}$ (Sasaki, 1990). Since a plausible value of $q$ is 2.5-2.75 from the present mass distribution, Ar abundance in gas-rich meteorites is explained when $f / r$ is larger than $10^{-2}\left[\mathrm{~m}^{-1}\right]$.

\section{Discussions}

In the later stage of solar system formation, gravitational scattering by massive Jupiter should enhance the inclination of planetesimals in the asteroidal region. If the vertical height of the disk there is larger than that of the inner regions, the trapped amount of noble gas should increase greatly. Therefore even if solar wind duration was shorter or intensity is weaker, a large amount of solar-type gas could be supplied to the dust (or larger bodies) in the asteroidal region.

In Venus, ratio of ${ }^{36}$ Ar mass to the total planetary mass is $2.4 \times 10^{-9}[\mathrm{~kg} / \mathrm{kg}]$, which is comparable to values of gas-rich meteorites with high noble gas abundances. The 
off-disk implantation can also supply Venus' solar-type noble gas if the dust with implanted gases accumulates onto the planet. During or after the implantation, both He and $\mathrm{Ne}$ should escape from grains because temperature there is higher than that in the asteroidal region. This may explain low $\mathrm{Ne} / \mathrm{Ar}$ of Venus.

Acknowledgments: The author thanks M. Ozima and T. Futagami for discussious. He is grateful to R. O. Pepin for valuable comments. Most parts of this work were done while the author stayed at University of Arizona. He thanks D. M. Hunten for supports and encouragements.

\section{References}

Goswani, J. N., Lal, D. and Wilkening, L. L. (1984) 'Gas-rich meteorites: probes for particle environment and dynamical processes in the inner solar system'; Space Sci. Rev. 37, 111-159.

Hayashi, C., Nakazawa, K., and Nakagawa, Y. (1985) 'Formation of the solar system', in D. C. Black and M. S. Matthews (eds.), Protostar and Planets II, The University of Arizona Press, 'Iucson, pp.1100-1153.

McElroy, M. B. and Prather, M. J. (1981) 'Noble gases in the terrestrial planets', Nature 15, 535-539.

Nakano, T. (1988) 'Formation of planets around stars of various masses - II. Stars of two and three solar masses and the origin and evolution of circumstellar dust cloud', Mon. Not. R. astr. Soc. 230, 551-571.

Pollack, J. B. and Black, D. C. (1982) 'Noble gases in planetary atmospheres: implications for the origin and evolution of atmospheres', Icarus 51, 169-198.

Prinn, R. G. and Fegley, B. Jr. (1989) 'Solar nebula chemistry: origin of planetary, satellite and cometary volatiles', in S. K. Atreya, J. B. Pollack, and M. S. Matthews (eds.) Origin and Evolution of Planetary and Satellite Atmospheres., The University of Arizona Press, Tucson, pp.78-136.

Sasaki, S. (1989) 'Penetration of the solar wind after dissipation of the solar nebula: origin of Venusian Ar by off-disk implantation of the solar wind', Proc. NIPR Symp. Antarct. Meteorites 2, 326-334.

Sasaki, S. (1991) 'Off-disk penetration of ancient solar wind', Icarus 90, in press.

Wetherill, G. W. (1981) 'Solar wind origin of ${ }^{36}$ Ar on Venus', Icarus 46, 70-80. 